\title{
Parasitóides (Hymenoptera) de dípteros coletados em fezes de galinha no sul de Goiás
}

\section{Parasitoids (Hymenoptera) of dipterous collected in manure chicken in south of Goias}

\author{
Carlos Henrique Marchiori ${ }^{1}$
}

\section{Resumo}

Este estudo teve como objetivo verificar os parasitóides associados com dípteros sinantrópicos em fezes de galinha, em Itumbiara, Estado de Goiás, de abril de 2006 a março de 2007. As pupas foram obtidas pelo método de flutuação. Elas foram individualizadas em cápsulas de gelatina até a emergência das moscas domésticas e/ou dos seus parasitóides. As espécies de parasitóides coletados foram: um espécime de Aphaereta sp. (Braconidae: Alysiinae), dois espécimes de Eurytoma sp. (Eurytomidae), 26 espécimes de Nasonia vitripennis (Walker, 1836) (Pteromalidae), 65 espécimes de Pachycrepoideus vindemmiae (Rondani, 1875) (Pteromalidae), 38 espécimes de Spalangia cameroni Perkins, 1910 (Pteromalidae), um espécime de Spalangia drosophilae Ashmead, 1887, 147 espécimes de Spalangia endius Walker, 1839, três espécimes de Spalangia nigra Latrielle, 1805, 10 espécimes de Spalangia nigroaenea Curtis, 1839, 21 espécimes de Spalangia sp. e 54 espécimes de Tachinaephagus zealandicus (Ashmead, 1904) (Encyrtidae). A espécie mais freqüente foi S. endius com 47,7\%.

Palavras-chave: Diptera, Hymenoptera, inimigo natural, controle biológico, microhimenópteros

\begin{abstract}
This study had as objective to verify the parasitoids associated with synanthropic dipterous in manure chicken, in Itumbiara, State of Goiás, from April 2006 to March 2007. The pupae were obtained by the flotation method. They were individually placed in gelatin capsules until the emergency of the adult flies or their parasitoids. The species of parasitoids collected were: one specimen of Aphaereta sp. (Braconidae: Alysiinae), two specimens of Eurytoma sp. (Eurytomidae), 26 specimens of Nasonia vitripennis (Walker, 1836) (Pteromalidae), 65 specimens of Pachycrepoideus vindemmiae (Rondani, 1875) (Pteromalidae), 38 specimens of Spalangia cameroni Perkins, 1910, (Pteromalidae), um specimen of Spalangia drosophilae Ashmead, 1887, 147 specimes de Spalangia endius Walker, 1839, three specimens of Spalangia nigra Latrielle, 1805, 10 specimens of Spalangia nigroaenea Curtis, 1839, 21 specimens of Spalangia sp. and 54 specimens of Tachinaephagus zealandicus (Ashmead, 1904) (Hymenoptera: Encyrtidae). The specie more frequent was $S$. endius with $47.7 \%$.
\end{abstract}

Key words: Diptera, Hymenoptera, natural enemy, biocontrol, microhimenopterous

1 Prof. do Centro Federal de Educação Tecnológica de Urutaí-UNED-Morrinhos. E-mail: chmarchiori@yahoo.com.br. 
Aves de galinha poedeira em granjas provocam acúmulo de esterco, um excelente substrato para a proliferação de moscas. Para controle destes insetos, um programa de manejo deve integrar métodos culturais, químicos e biológicos. Os agentes de controle biológico terão maior chance de serem bem sucedidos se forem mais adaptados à região, à época do ano e ao tipo de esterco do local (COSTA; BERTI FILHO; SILVEIRA NETO, 2004).

Os parasitóides são agentes responsáveis pela redução das populações de moscas que proliferam em esterco (MARCHIORI; OLIVEIRA; LINHARES, 2001), cadáveres e carcaças de animais. Além disso, sendo inimigos naturais de pragas agrícolas, podem ser usados em programas de controle biológico (SCATOLINI; DIAS, 1997).

O objetivo deste trabalho é relatar os parasitóides de dípteros sinantrópicos coletados em um aviário no Sul de Goiás.

O experimento foi realizado na granja da Chácara Casa do Campo em Itumbiara ( $\left(8^{\circ} 25^{\prime} \mathrm{S}\right.$ e $\left.49^{\circ} 13^{\prime} \mathrm{W}\right)$, Goiás. As fezes coletadas originaram-se de 40 aves da linhagem "Hyline" criadas no sistema de gaiolas. As fezes acumuladas sob as gaiolas variavam de umidade, de pastosa a firme. As fezes frescas (pastosa), coletadas imediatamente após a emissão, foram colocadas em cinco bacias de $30 \mathrm{~cm}$ de diâmetro por $12 \mathrm{~cm}$ de altura, que foram deixadas no próprio ambiente, em local seco, por 15 dias; para a extração das pupas pelo método da flutuação, alterando-se os procedimentos de rotina para este tipo de experimento. Os parasitóides e as moscas emergidos foram identificados com auxílio de um microscópio estereoscópio.

A porcentagem total de parasitismo foi calculada através do número de pupas parasitadas/número total de pupas coletadas x 100. A porcentagem de parasitismo de cada espécie de parasitóide foi calculada através do número de pupas parasitadas por cada espécie de parasitóide/número total de pupas daquele hospedeiro x 100. A identificação dos parasitóides foi realizada conforme Rueda e Axtell (1985). Os espécimes de parasitóides foram depositados no Laboratório do Instituto Luterano de Ensino Superior de Itumbiara, Goiás.

Foram coletados 3086 dípteros sinantrópicos, dos quais originaram 368 parasitóides de 311 pupários (Tabela 1). As espécies de parasitóides coletados foram: um espécime de Aphaereta sp. (Braconidae: Alysiinae), dois espécimes de Eurytoma sp. (Eurytomidae), 26 espécimes de Nasonia vitripennis (walker, 1836) (Pteromalidae), 65 espécimes de Pachycrepoideus vindemmiae (Rondani, 1875) (Pteromalidae), 38 espécimes de Spalangia cameroni Perkins, 1910 (Pteromalidae), um espécime de Spalangia drosophilae Ashmead, 1887, 147 espécimes de Spalangia endius Walker, 1839, três espécimes de Spalangia nigra Latrielle, 1805, 10 espécimes de Spalangia nigroaenea Curtis, 1839, 21 espécimes de Spalangia sp. e 54 espécimes de Tachinaephagus zealandicus (Ashmead, 1904) (Encyrtidae). A espécie mais coletada foi $S$. endius com $47,6 \%$. A capacidade de busca do parasitóide, a sazonalidade, a maior presença de seus hospedeiros na área de coleta e o seu maior potencial competitivo na fase larval pode explicar a maior freqüência dessa espécie, apesar não tenha sido testado nenhum fator para explicar a diferença na freqüência dos parasitóides neste trabalho. Spalangia endius é um parasitóide cosmopolita, sendo capaz de atacar pupas de Calliphoridae, Sarcophagidae, Muscidae e Tephritidae (BLUME, 1970; SERENO; NEVES, 1993). 
Tabela 1. Parasitóides himenópteros de dípteros sinantrópicos coletados em fezes de galinhas poedeiras em Itumbiara, Goiás, Brasil.

\begin{tabular}{|c|c|c|c|c|}
\hline Diptera & $\begin{array}{l}\mathrm{N}^{\mathrm{o}} \cdot \mathrm{de} \\
\text { indivíduos }\end{array}$ & Parasitóides & $\begin{array}{c}\text { Pupas } \\
\text { parasitadas }\end{array}$ & Porcentagem \\
\hline \multicolumn{5}{|l|}{ Fanniidae: } \\
\hline $\begin{array}{l}\text { Fannia pusio } \\
\text { (Wiedemann) }\end{array}$ & 153 & Eurytoma sp. & 2 & 1,3 \\
\hline Muscidae: & & $\begin{array}{l}\text { Pachycrepoideus vindemmiae } \\
\text { (Rondani) }\end{array}$ & 9 & 5,9 \\
\hline \multirow[t]{9}{*}{ Musca domestica L. } & 1982 & Aphaereta sp. & 1 & 0,1 \\
\hline & & Nasonia vitripennis (Walker) & 11 & 0,5 \\
\hline & & Pachycrepoideus vindemmiae & 52 & 2,6 \\
\hline & & Spalangia cameroni Perkins & 38 & 1,9 \\
\hline & & Spalangia drosophilae Ashmead & 1 & 0,1 \\
\hline & & Spalangia endius Walker & 143 & 7,2 \\
\hline & & Spalangia nigra Latrielle & 3 & 0,2 \\
\hline & & Spalangia nigroaenea Curtis & 10 & 0,5 \\
\hline & & Spalangia sp. & 21 & 1,1 \\
\hline \multirow[t]{2}{*}{ Ophyra sp. } & 529 & $\begin{array}{l}\text { Tachinaephagus zealandicus } \\
\text { (Ashmead) } \\
\text { Nasonia vitripennis }\end{array}$ & $\begin{array}{l}1 \\
1\end{array}$ & $\begin{array}{l}0,1 \\
0,2\end{array}$ \\
\hline & & Spalangia endius & 4 & 0,8 \\
\hline \multicolumn{5}{|l|}{ Sarcophagidae: } \\
\hline \multirow{2}{*}{$\begin{array}{l}\text { Sarcodexia lambens } \\
\text { (Wiedemann) }\end{array}$} & 222 & Pachycrepoideus vindemmiae & 2 & 0,9 \\
\hline & & Tachinaephagus zealandicus & 9 & 4,1 \\
\hline \multicolumn{5}{|l|}{ Syrphidae: } \\
\hline $\begin{array}{l}\text { Ornidia obesa } \\
\text { Fabricius }\end{array}$ & 226 & Pachycrepoideus vindemmiae & 2 & 0,9 \\
\hline $\begin{array}{l}\text { Outras espécies } \\
\text { identificadas não } \\
\text { parasitadas }\end{array}$ & 04 & - & - & - \\
\hline Total & 3116 & - & 308 & - \\
\hline
\end{tabular}


A redução da população de moscas, que proliferam no esterco de galinhas encontrado neste estudo, foi de 10,0\% (porcentagem de parasitismo). A porcentagem de parasitismo apresentada pelas espécies Aphaereta sp., Eurytoma sp., $N$. vitripennis, $P$. vindemmiae, $S$. cameroni, $S$. drosophilae, S. endius, S. nigra, S. nigroaenea, Spalangia sp. e T. zealandicus foi de $0,1 \%, 0,1 \%$, $0,8 \%, 2,1 \%, 1,2 \%, 0,1 \%, 4,7 \%, 0,1 \%, 0,3 \%, 0,7 \%$ e $1,7 \%$, respectivamente.

A espécie que apresentou maior porcentagem de parasitismo foi S. endius no hospedeiro Musca domestica L., 1758 (Diptera: Muscidae) com 7,2\%, provavelmente devido a maior afinidade pelas espécies hospedeiras presentes, apesar que não tenha sido testado nenhum fator para explicar a diferença na porcentagem de parasitismo dos parasitóides.

A avicultura, no Sul do Estado de Goiás, é uma atividade econômica importante e o estudo de métodos alternativos de controle, como os microhimenópteros parasitóides que são naturalmente coletados emergindo de pupários de dípteros. Estes proliferam no esterco de aves e podem ser responsáveis pelo desenvolvimento sustentável para a região estudada, inclusive com a utilização de algumas espécies como controladores biológicos.

\section{Referências}

BLUME, R. B. Insects associated with bovine droping in kerr and bexar county, Texas. Journal of Economic Entomology, Lanham, v. 63, n. 3, p. 1023-1024, 1970.

COSTA, V. A.; BERTI FILHO, E.; SILVEIRA NETO, S. Parasitóides (Hymenoptera: Chalcidoidea) de moscas sinantrópicas (Diptera: Muscidae) em aviários de Echaporã, SP. Arquivos do Instituto Biológico, São Paulo, v. 71, n. 2, p. 203-209, 2004.

MARCHIORI, C. H.; OLIVEIRA, A. T.; LINHARES, A. X. Artrópodes associados a massas fecais bovinas no $\mathrm{Sul}$ do Estado de Goiás. Neotropical Entomology, Londrina, v. 30, n. 1, p. 19-24, 2001.

RUEDA, L. M.; AXTELL, R. C. Guide to common species of pupal parasites (Hymenoptera: Pteromalidae) of the house fly and other muscoid flies associated with poultry and livestock manure. North Carolina Agricultural Research Service, Raleigh, v. 278, p. 1-88, 1985. Technical Bulletin.

SERENO, F. T. P. S.; NEVES, D. Microhimenópteros (Pteromalidae) parasitóides de Diptera (Muscidae, Otitidae) em uma granja de bovinos em Igarapé, Estado de Minas Gerais, Brasil. Revista Brasileira de Entomologia, Curitiba, v. 37, n. 3, p. 563-567, 1993.

SCATOLINI, D.; DIAS, A. M. P. A fauna de Braconidae (Hymenoptera) como bioindicadora do grau de preservação de duas localidades do Estado do Paraná. Revista Brasileira de Ecologia, Rio Claro, v. 1, p. 84-87, 1997. 